\title{
The Schröder staircase: A new perspective
}

\author{
J. P. HARRIS \\ Brain and Perception Laboratory, Department of Anatomy, The Medical School \\ Bristol BS8 1TD, England
}

\begin{abstract}
A range of variants of the Schröder staircase was generated by computer, giving quantifiable changes of perspective and the option of adding appropriate texture to the steps of the staircase. Increases in perspective and the addition of texture gradients led to increases in the proportion of viewing time during which the percept appropriate to the perspective was reported. It is suggested that this might be a useful method of studying the processing of depth information.
\end{abstract}

There are several reasons for supposing that the perceptual alternations of a class of reversible figures, which includes the Necker cube, Schröder staircase, and Mach card, are linked with depth processing mechanisms. First, subjects often describe such an alternation as a reversal of the apparent depth relationships within the figure. Secondly, adding depth cues to such figures can alter both the rate of alternation and the relative durations of the various percepts. A third line of evidence is much more indirect: although the alternation rate of a number of multistable figures changes with the subjects' viewing distance, that of depth reversible figures does not (Atkinson, Campbell, Fiorentini, \& Maffei, 1973).

The evidence that depth cues affect reversible figures is somewhat variable in its quality, ranging from brief anecdotal reports, through incidental observations, to experiments in which attempts were made to quantify both the depth information added to the reversible figure and the resulting changes in perceptual alternation. For example, Ogle (1962) mentions that the addition of perspective to the Necker cube and Schröder staircase produces a tendency to one percept. Orbach and Zucker (1965) report that removing those edges from a Necker cube which would be hidden if it were solid has a similar effect. It was found by Howard (1961) and Virsu (1975) that adding disparity to a skeletal wire cube and Schröder staircase, respectively, increases the initial period before a perceptual reversal. In a study of the Necker cube, Cormack and Arger (1968) reported that increases in disparity up to $45 \mathrm{~min}$ of arc both increased the durations of the percepts appropriate to the disparity and decreased the alternation rate, but that a further increase to $90 \mathrm{~min}$ of arc had little additional effect. This last study, if

I thank the MRC for support; Nick Markovits for his programs, advice, and help, without which the study would have been impossible; Ian Low for building the timers and printer; Philip Clark for photography; and Richard Gregory, David Rose, and Priscilla Heard for helpful comments. not unique, must be one of the few investigations in which the added depth cue has been systematically varied.

These studies are usually said to tell us something about perceptual multistability. But it also seems plausible to argue that they tell us something about the way in which depth information is processed by the visual system. There are some assumptions necessary for this argument, which will now be considered. The first is that the kinds of perceptual computations and decisions made when viewing multistable figures are not different from those made when viewing other kinds of pictures and real scenes. Thus, perception is seen as rather like a problemsolving process, in which evidence for various solutions is accumulated. Once evidence for a particular solution (or percept) exceeds some value, that is accepted, but the search for other solutions continues. From time to time, evidence for alternative solutions will reach some threshold and be accepted in turn. A model of this kind which incorporates perceptual alternation into more general perceptual processing has been proposed by Vickers (1972a, 1972b). It seems fairly easy to make such a model give a high alternation rate for ambiguous figures, with a decreasing alternation rate or increased duration of the appropriate percept when depth cues are added, and a much reduced or zero alternation rate for real scenes, at least when these contain multiple depth cues.

The second assumption is that depth cues like perspective or disparity are processed by the visual system in a similar way, whether they are carried by reversible figures or not. So the degree of convergence of two lines, or the horizontal disparity of two features, carries the same depth information in isolation as it does as part of a suitably treated Necker cube. These two assumptions are parsimonious; they suggest that reversible figures do not "call up" perceptual processes which would otherwise be dormant, and that depth cues are processed similarly whatever their carrier stimulus. 
A third assumption is that the frequency and duration of percepts not favored by depth cues added to a reversible figure will be some function of the salience of those cues. So, for example, the size of a binocular disparity will be reflected in some way in the accumulation of evidence for one percept, larger disparities making larger contributions than small ones. The nature of the function relating depth information to this hypothetical accumulation of sensory evidence, and hence to the measured frequency and duration of particular percepts, could be determined experimentally. In the present study, an attempt was made to measure the strength of two perspective cues by seeing how effective they were at biasing the perception of an otherwise ambiguous figure, the Schröder staircase.

The visual processing of perspective information is not usually examined in this way. Such cues have been studied by presenting the subject with, say, a texture gradient on a vertical or horizontal surface and having him match its apparent slant with an adjustable tilting rod (Clark, Smith, \& Rabe, 1956), or compare it with the apparent slant of a neighboring textured surface (Phillips, 1970). Alternatively, the subject may be asked to set a marker at the apparent midpoint of such a gradient (Newman, 1971). It could be argued that some of these tasks have intellectual, memory, or motor components which make them less than ideal measures of the subject's immediate percept. Judgments of the apparent orientation of an ambiguous figure, on the other hand, although presumably based on similar perceptual computations do not seem to require conscious assessments of amounts of slant or depth. This method of evaluating depth cues may be a useful complement to those described above.

The reversal rate of ambiguous figures has already been studied as a function of a variety of variables such as the personality (Frederiksen \& Guildford, 1934) or fatigue (Smith, 1916) of the subject, the length of the observation period (Brown, 1955; Price, 1969), or the size, color, area, or brightness of the figure (Graham, 1929; Washburn, Malloy, \& Naylor, 1931). In the present experiments, subjects were presented with a range of variants of the Schröder staircase, which differed in their perspective convergence and in whether or not they had additional consistent texture gradients on their steps. The proportion of the total viewing period was measured during which the subject's percept was appropriate to the perspective of the stimulus figure. Figures of this general kind have already been investigated by Hagen and Elliot (1976), who used a system described by Reggini (1975) to produce drawings of a number of simple geometrical shapes such as cubes. The perspective of the drawn figures varied between the normal (or, as Reggini calls it, “conical”) projection, and the axonometric, or zero, perspective. Hagen and Elliot asked their subjects to rate how natural or accurate each drawing seemed, and found a consistent preference for the axonometric stimuli, with a linear decrease from axonometric to "normal" perspective. Although the experiments were different, that study was similar to the one reported here in the way in which the stimuli were generated and an attempt made to quantify their perspective.

\section{EXPERIMENT 1}

\section{Method}

Stimuli. All stimuli were variants of a five-tread staircase whose zero perspective version is shown in Figure 1 (lower right staircase). They were drawn by a computer under the control of a graphics package developed for computer aided design." The program stores in its memory a three-dimensional representation of the staircase, which can be drawn from a specifiable point of view, in "pinhole camera" perspective. That is, the "projection rays" from the object to the viewpoint are straight. The ratio of the dimensions, tread depth: riser height: tread width: total height was 1:1:2:5. The computer's "viewpoint" lay along a line projecting from the center of the intersection of the tread and riser of the central step. This line was at $45^{\circ}$ to the plane of the "stairtreads," the "stair risers," and the "walls" of the "stairwell." One independent variable in this study was the notional distance along the line of sight of the computer's "point of view." Expressed as multiples of the total staircase height, the range of distances was $2,4,6,9,12$,
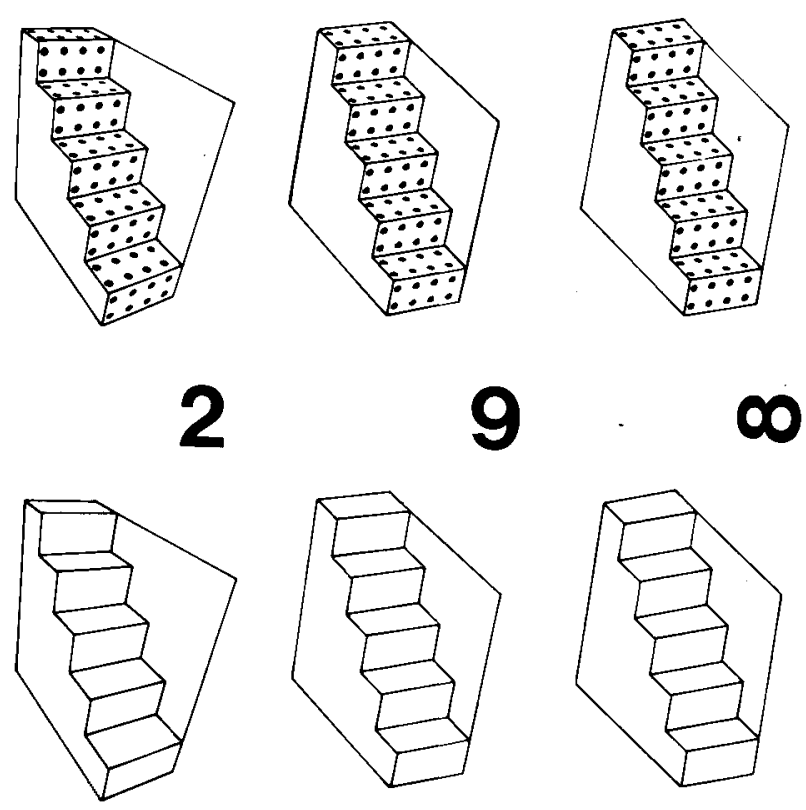

Figure 1. Examples of the stimuli used in the present experiments, together with the distance from the staircase (expressed as a multiple of its height) of the computer's notional viewpoint. The only difference between the upper and lower rows of stimuli is the addition of texture appropriate to the perspective in the upper row. The right-hand pair of stimuli are versions of the familiar zero perspective Schröder staircase. See text for further details. 
15, and an infinite viewing distance. As notional viewing distance is increased, so the amount of perspective convergence decreases, and at an infinite viewing distance is zero, hence giving the familiar Schröder staircase. The computer always made its drawings the same overall size, expanding them equally in $x$ and $y$ until they just fitted its fixed output format. The second variable was the presence or absence of texture on the treads and risers of the staircase. This consisted of two rows of four equispaced circles on each tread and riser, drawn as appropriate to the particular viewing distance of the computer. The lines in the computer hard copy were thickened up using a drawing pen, and the circles were filled in to give disks. The resulting pictures were photographed on high-contrast film and mounted as slides for conventional projection. (See examples in Figure 1, which shows negatives of the experimental slides).

Apparatus. The stimulus slides were back-projected on a frosted perspex (Plexiglas) screen, $12^{\circ} 30^{\prime}$ wide $\times 10^{\circ} 50^{\prime}$ high, which was itself inset in the center of an opaque matt black card surround $29^{\circ}$ wide $\times 19^{\circ} 30^{\prime}$ high. A Leitz Pradovitz automatic projector was used. Slide presentation time was governed by an electromechanical iris shutter controlled by an electronic timer. An electronic counter summed the time intervals during which a push-button switch was held closed. When the timer closed the shutter, thus terminating the slide presentation, it also caused the summed time on the counter to be printed out, together with a slide identification number set with thumb wheel switches by the experimenter. When projected, the size of the staircase figures fell within an imaginary rectangle whose height was always $10^{\circ}$ and whose width varied between $7^{\circ}$ and $7^{\circ} 30^{\prime}$. Line thickness in the figure was about $5^{\prime}$.

The subject sat at a distance of $135 \mathrm{~cm}$ from the screen, which, to reduce sensory evidence that it was really flat, was viewed monocularly. (Subjects decided at the beginning of the experiment which eye they would prefer to use to view the patterns, and the other eye was covered with an eyepatch. The subjects wore their normal optical corrections for the viewing distance). It should be noted that the perspective of the stimuli drawn from a notional computer viewing distance of 9 times the staircase height was appropriate to the subjects' viewing distance. A circular fixation spot (size $25^{\prime}$ ) was provided at the center, of the display. The subject was asked not to fixate the center of the spot, but to let his eye roam over it and around its perimeter. The staircase figures were projected in the orientation shown in Figure 1, having been adjusted in their slide mounts so that the center of the intersection of the tread and riser in the central step fell in the middle of the fixation spot. The subject's seat was arranged so that his eye was on the optical axis of the projector. The projection screen was normal to this axis.

Measured with an SEI spot photometer, the brightness of the lines and disks in the patterns on the screen was about $300 \mathrm{~cd} \mathrm{~m}^{-2}$, that of the ground about $15 \mathrm{~cd} \mathrm{~m}^{-2}$. The only lighting in the experimental room was provided by scattered light from the projector and a small dim lamp by whose light the experimenter manipulated the apparatus and made notes. Neither of these sources was directly visible to the subject.

Procedure. In a single experimental session, which lasted about $1 / 2 \mathrm{~h}$, each of the 14 stimulus slides $(7$ different amounts of perspective with and without texture) was seen once by each subject. The sole exception to this rule was that the axonometric version of the staircase without texture-that is, the normal Schröder staircase figure-was used in a short practice session, during which instructions were given for the main experiment. After the subject had put on the eye patch and had his seating adjusted, this slide was projected on the screen. The reversal phenomenon was noted by and discussed with the subjects. It was emphasized that the subjects were not to try to make the figures reverse; rather, they were to sit there in a passive mode and let the alternations take their course. Two kinds of percept were agreed and defined with the subject: on the one hand, the percept of a set of stairs, as it were, seen from above; on the other, a "default" category of percepts which was usually the underside view of a set of stairs, but could include any other possible percept, such as the display appearing flat or as a series of arrowheads, and so on. The subject was then handed the push-button switch and instructed on its use. Half the subjects were instructed to press the button and hold it down during the first category of percept, the other half during the "default" category. The room lights were then extinguished, and the subject was allowed between 1 and 2 min practice to observe the figure and record changes in his perception of it. Any necessary clarification of the instructions was made, and the experiment proper began.

Each of the 14 stimulus slides was presented to each subject in a different pseudorandom order. Presentation time in all cases was $100 \mathrm{sec}$. Ten subjects (seven males, three females) with no known visual defects took part in the experiment.

\section{Results}

The results are shown in Figure 2. Here the percentage of the viewing time in which the stairs were seen as from above-the percept appropriate to the perspective in the drawing - is plotted against a measure of that perspective. The numbers on the abscissa are multiples of the staircase height; they represent the distance from the staircase of the computers' notional viewpoint when drawing the staircase. They can be used as a convenient scale on which to measure perspective.

The first point to notice in these results is that there does indeed appear to be a simple relationship between the amount of perspective and the proportion of the viewing time in which the appropriate percept is reported. It falls linearly from over $70 \%$ for the

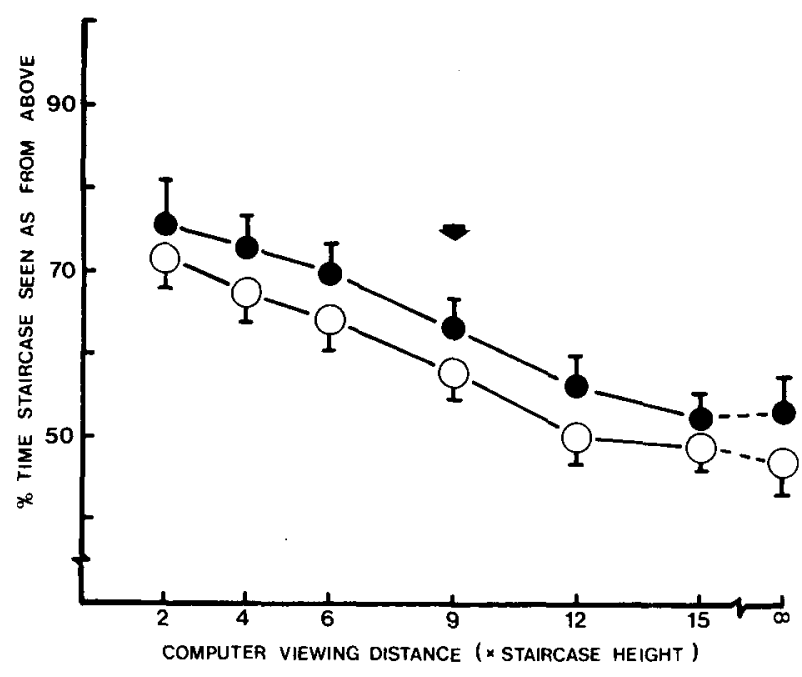

Figure 2. Relationship found in Experiment 1 between the perspective of the stimulus and the percentage of the viewing period during which the percept appropriate to the perspective was reported. Filled circles represent means from stimuli with texture, open circles, means from plain stimuli. Vertical bars represent standard errors. Arrow shows stimuli whose perspective was appropriate to the subjects' viewing distance. See text for further details. 
pictures with the greatest amount of perspective to around $50 \%$ for the pictures with the least. Notice also that the zero perspective, or axonometric figures, drawn as from an infinite viewing distance, produce results which are very similar to the latter. An analysis of variance showed that the main effect of varying perspective was significant $[F(6,54)=20.03, p<.001]$.

The second major feature of the results demonstrates an effect of adding texture to the stimuli. The two curves (for stimuli with and without texture) appear to be essentially parallel. Thus, whatever the perspective in the outline figure, the addition of appropriate texture causes an increase of some $5 \%$ in the amount of total viewing time in which the appropriate percept is reported. From the analysis of variance, the main effect of adding texture was significant $[F(1,9)$ $=8.56, \mathrm{p}<.025]$.

\section{Discussion}

One finding which seems of value in the present experiment is methodological. The consistent changes in report found for consistent changes in the stimuli suggest that this may indeed be a useful technique for investigating the processes underlying depth perception. It might be used to assess, for example, the relative salience of varieties of texture or other ways of indicating the planes between edges and corners.

The experiment has also produced two observations which seem to be of some theoretical interest. First, it seemed possible that when the perspective was appropriate to the subject's viewing distance from the screen, the display might be particularly stable, with the percept appropriate to the perspective being reported for most of the viewing time. However, no such effect was noted. There are no discontinuities in the graphs at a computer viewing distance of 9 times the staircase height, although the perspective in those displays was appropriate to the subject's viewing distance.

Secondly, the results demonstrate a clear effect of adding texture to the steps, although none of the differences between the means of the pairs of stimuli at each perspective reached significance at the $5 \%$ level in Newman-Keuls comparisons. It appears that, for pictures with greater than zero perspective, the redundant depth information may be combined in some simple way with that from the outlines. However, an apparent difficulty for this view is that the difference between figures with and without texture is maintained even for the axonometric figures. Notice that there are no gradients of texture within the planes of the treads or risers in these figures, and so the texture is unable to suggest that a particular intersection is nearer or further away than its neighbor. This experiment leaves open the possibility that the effect of adding texture gradients to the stairs is not, per se, to bias the visual system towards one perspective interpretation. Some subjects noted that the stimulus figures did not always appear threedimensional. Perhaps the added texture somehow prevented the pictures from appearing flat. The bias towards seeing staircases from above rather than from below might then be produced by the subjects' greater experience of seeing them from this aspect.

Because of the theoretical importance of the presence or absence of a difference between the zeroperspective stimuli with and without texture, it seemed important to establish how robust these effects were. Because of the large number of stimuli, it was difficult to counterbalance properly against order effects in Experiment 1. Similarly, a range effect may have been operating, so that an increase in reporting "stairs from above" for textured stimuli whose perspective was appropriate was continued for the zero-perspective stimuli. For these reasons, the number of stimuli was reduced in a second experiment.

Some subjects reported that some stimulus figures seemed to reverse in aspect more readily than others. It seemed worthwhile to check on this by measuring the frequency of reversals for two reasons. First, this measure might throw light on the nature of the mechanism underlying perspective reversals. For example, if reversal rate remained constant, despite changes in the perspective of the stimulus and the resulting change in perspective-appropriate perception, it could suggest that switching rate reflected some "free-running" process. If, on the other hand, frequency of alternation decreased as the perspective of the stimulus became more exaggerated, then it would appear that the switching rate as well as the durations of the alternating percepts could be influenced by visual processes detecting texture or orientation gradients or underlying their perception as depth cues. Secondly, if the interval between reversals was long, compared with the viewing period of $100 \mathrm{sec}$, then misleading results might have been obtained. (To exaggerate, if the average interval was $50 \mathrm{sec}$, then a viewing period of $60 \mathrm{sec}$ might give a different picture from a viewing period of $200 \mathrm{sec}$ ). Measuring reversal frequency and increasing viewing time seemed useful precautions against this.

\section{EXPERIMENT 2}

\section{Method}

The apparatus and procedure were the same as in Experiment 1, with the following exceptions. In addition to the counter which summed the time for which the push button was held down, a further counter was added which counted the number of times the button was pushed. The stimulus set was reduced to six slides. Expressed as multiples of the picture height, these corresponded to computer viewing distances of 2,9 , and infinity, only one of the two slides at each viewing distance having added texture. Viewing time was increased to $120 \mathrm{sec}$. In order to guard against any effects of prior exposure to one of the experimental slides, the 
instructions were given and the subject allowed to practice with the slide drawn from a computer notional viewing distance of 15 times the picture height. This was not included in the experimental set. Rather than allowing the subjects to choose with which eye they would view the experimental stimuli-as was done in Experiment 1 -the dominant eye (as indicated by a sighting task) was always used. Sixteen subjects-seven males and nine females -took part in the experiment. None of them had taken part in experiments with stimuli of this type before and they all wore any optical correction which they would normally wear for the viewing distance of $135 \mathrm{~cm}$. Although it would not have been possible to counterbalance the order of presentation of the slides without using a much larger sample of subjects, a different order of presentation was used for each subject and these orders chosen so that each slide occupied a given serial position a similar number of times.

\section{Results}

The results are plotted in Figure 3. Figure $3 \mathrm{a}$ is exactly analogous to Figure 2 . The average duration of the perspective-appropriate percept is plotted against the perspective of the staircase. The data show two effects which were also apparent in Figure 2. First, the duration of the appropriate percept falls with the perspective of the staircase; secondly, for computer viewing distances of twice and nine times the picture height, there is a clear effect of adding texture. It looks as though texture adds a roughly constant amount (about $8 \%$, in this experiment) to the duration of the appropriate percept. However, this effect did not occur for the axonometric stimuli, the means and standard errors of the plain and textured stimuli being almost identical. These apparent effects were confirmed by the analysis of variance: both the effects of perspective change $[F(2,15)=33.32, p<.001]$ and the effects of adding texture $[F(1,15)=9.40, p<.01]$ were significant. However, subsequent Newman-Keuls comparisons between pairs of means showed that the significance of this effect was confined to those stimuli with perspective. The differences between plain and textured stimuli drawn from viewing distance $2(p<.01)$ and viewing distance $9(\mathrm{p}<.05)$ were significant, but the very small difference $(.3 \%)$ between the zero perspective stimuli was not.

Figure $3 \mathrm{~b}$ shows the mean number of reversals per minute plotted against stimulus perspective. It can be seen that the means for the plain and textured stimuli are almost identical for each perspective. The small differences did not reach significance in the analysis of variance $[F(1,15)=.9]$. However, there is a lower alternation rate for the drawings with the most exaggerated amount of perspective, compared with the other stimuli, for which the means are almost identical. The analysis of variance confirmed this impression $[F(2,30)=10.64, p<.001]$, and Newman-Keuls comparisons showed that the significance of the effect was confined to the differences between the stimuli with the most exaggerated perspective and the others. None of the small differences between any of the
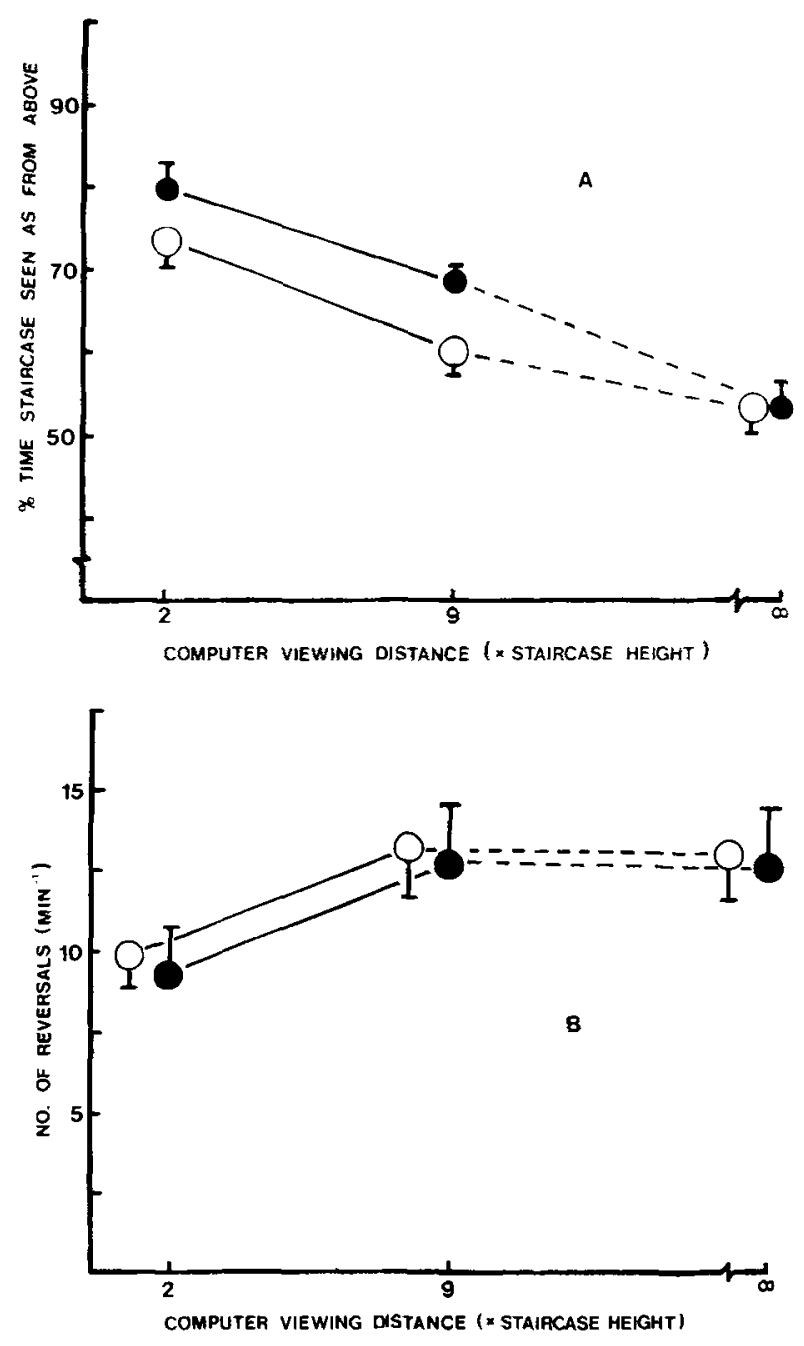

Figure 3. (A) Relationship found in Experiment 2 between the perspective of the stimulus and the percentage of the viewing period during which the percept appropriate to the perspective was reported. Other conventions as for Figure 2 . The extreme righthand open circle has been moved slightly to the left to avoid overlap. See text for further details. (B) Relationship found in Experiment 2 between the perspective of the stimulus and the reported mean number of perceptual reversals. Other conventions as for Figure 2. Open circles have been moved slightly to the left to avoid overlap. See text for further details.

means for staircases drawn from 9 times the picture height or from infinity approached significance. Notice also that the average interval between reversals was about 5 or $6 \mathrm{sec}$. Since this is short compared with the viewing period, it seems unlikely that the results were biased by some form of sampling error.

\section{Discussion}

Experiment 2 confirmed two aspects of the results of Experiment 1, namely the increase in the duration of the appropriate percept as perspective convergence is increased and the constant effect of appropriate texture on the stimuli with perspective. It was argued 
in the introduction that such effects may throw light on the visual processing of depth information. It is interesting, therefore, to find that Clark, Smith, and Rabe (1955) reported that increasing the convergence of the edges of an outline form produced increases in its perceived slant in depth. The same authors (1956) looked at the effect of adding texture to such an outline form. Although it was small and insignificant, there was an increase in perceived slant, as would be predicted from the present account. The present findings also support the general approach to perceptual alternations suggested by Vickers (1972b).

An important finding from Experiment 2 was the absence of any difference between the results from the plain and textured axonometric stimuli. Had a difference persisted under the rather better controlled conditions of Experiment 2, it would have suggested that the increase in time occupied by the appropriate percept was not due to the perspective information carried by the texture gradients, but perhaps to a less interesting incidental effect of adding texture, such as a change in luminance. This interpretation is not supported by the data of Experiment 2.

The frequency results obtained in Experiment 2 suggest that as perspective is increased, the rate of alternation does not initially decrease. Rather, the durations of appropriate percepts become longer, with corresponding shortening of inappropriate percepts. But as perspective becomes more exaggerated, so the alternation rate starts to decrease. The probability of trying a new interpretation of the stimulus seems at least partly a function of the likelihood of the present interpretation being correct.

In both experiments, the figures whose perspective was correct for the subjects' viewing position were not found to be the most stable. This finding may be related to those summarized by Hagen (1978), who concludes that when subjects are making judgments about size and distance relationships within pictures, their performance is no better, and may even be worse, when they view a picture from the correct station point. Notice, however, that in Figure 2, the duration scores from the figures correct for the subjects' viewing distance were what would be expected if only their perspective were influencing their perception. In this experiment, station point seems to have been irrelevant. Perhaps particular tasks, displays, and deviations from the correct point are necessary to show up such station point effects.

A possible shortcoming in the generation of the staircase pictures should also be noted. It includes the assumption that to faithfully represent lines and edges which are straight in the real world, they should also be straight in the drawing. This assumption is probably only approximately correct. Reggini's (1975) program, referred to earlier, which permits con- trolled deviations from straight lines, was devised to correct apparent distortions of perspective in architects' drawings. Battro, Reggini, and Karts (1978) and Finch (1977) are among the latest to report experiments and demonstrations which support the idea that visual space is curved. However, it seems safe to say that any errors in the figures from this source would be small.

In summary, then, the quantitative measurement of their ability to prevent perceptual reversals of ambiguous figures may be a useful way of assessing the effects of some depth cues. Since depth cues can be combined in ways which approximate those of real-world stimuli, some of the doubts which must accompany such unnatural techniques as putting one depth cue in conflict with another can be avoided. It will be of interest therefore to see whether, despite its obvious limitations, the technique can distinguish the effects of other perspective or stereoscopic cues.

\section{REFERENCES}

Atkinson, J., Campbell, F. W., Fiorentini, A., \& Maffei, L. The dependence of monocular rivalry on spatial frequency. Perception, 1973, 2, 127-133.

Battro, M., Reggini, H. C., \& Karts, C. Perspectives in open spaces: A geometrical application of the Thouless index. Perception, 1978, 7, 583-588.

Brown, K. T. Rate of apparent change in a dynamic ambiguous figure as a function of observation time. American Journal of Psychology, 1955, 68, 358-371.

Clark, W. C., Sмith, A. H., \& Rabe, A. Retinal gradient of outline as a stimulus for slant. Canadian Journal of Psychology, 1955, 9, 247-253.

Clark, W. C., Smith, A. H., \& RaBe, A. The interaction of surface texture, outline gradient, and ground in the perception of slant. Canadian Journal of Psychology, 1956, 10, 1-8.

Cormack, R. H., \& ARgER, R. Necker cube perspective dominance as a function of retinal disparity. Perceptual and Motor Skills, 1968, 26, 367-370.

Finch, D. Hyperbolic geometry as an alternative to perspective for constructing drawings of visual space. Perception, 1977 , 6, 221-225.

Frederiksen, N. O., \& Guildford, J. P. Personality traits and fluctuations of the outline cube. American Journal of Psychology. $1934,46,470-474$.

Graham, C. H. Area, color and brightness difference in a reversible configuration. Journal of General Psychology, 1929, 2, 470-481

HAGEN, M. A. An outline of an investigation into the special character of pictures. In H. E. Pick \& E. Saltzman (Eds.), Modes of perceiving and processing information. Hillsdale, N.J: Erlbaum, 1978.

HaGEN, M. A., \& Elliot, H. B. An investigation of the relationship between viewing conditions and preference for time and modified perspective in adults. Journal of Experimental Psychology, 1976, 2, 479-490.

How ARD, I. P. An investigation of a satiation process in reversible perspective of revolving skeletal shapes. Quarterly Journal of Experimental Psvchology, 1961, 13, 19-33.

Newman, C. V. The influence of visual texture density gradients on relative distance judgements. Quarterly Journal of Experimental Psychologv, 1971, 23, 225-233.

OGil., K. N. Perception of distance and size. In H. Davson 
(Ed.), The eye. (Vol. 4) Visual optics and the optical space sense. New York: Academic Press, 1962.

OraAch, J., \& Zucken, E. Reversibility of the Necker cube: VI. Effects of interpolating a non-reversing cube. Perceptual and Motor Skills, 1965, 20, 470-472.

Philltps, R. J. Stationary visual texture and the estimation of slant angle. Quarterly Journal of Experimental Psychology, $1970,22,389-397$.

Price, J. R. Effect of extended observation on reversible perspective duration. Psychonomic Science, 1969, 16, 75-76.

Reggini, H. C. Perspective using curved projection rays and its computer application. Leonardo, 1975, 8, 307-312.

Smith, M. A contribution to the study of fatigue. British Journal of Psychology, 1916, 8, 327-350.

ViCkers, D. Decision processes in perceptual organization. In J. F. O'Callaghan (Ed.), Pictorial organization and shape. Canberra, A.C.T: Division of Computing Research C.S.I.R.O., 1972. (a)
VICKERS, D. A cyclic decision model of perceptual alternation. Perception, 1972, 1, 31-48. (b)

Virsu, V. Determination of perspective reversals. Nature, 1975, 257, 786-787.

Washburn, M. F., Malloy, H., \& Naylor. A. The influence of the size of an outline cube on the fluctuations of its perspective. American Journal of Psychology, 1931, 43, 484-489.

\section{NOTE}

1. The package is called GINO-F (Graphical INput/OutputFortran version), and is used at Bristol under license from its originators: The Computer Aided Design Centre, Madingley Road, Cambridge, CB3 OHB, England.

(Received for publication February 27, 1979; revision accepted August 22, 1979.) 